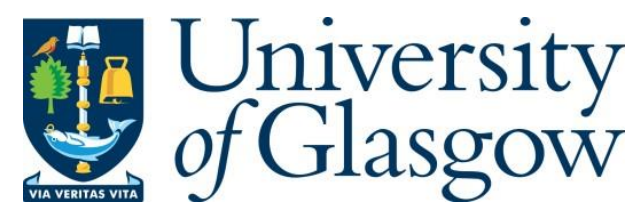

Alharbi, K. H., Khalid, A., Ofiare, A., Wang, J., and Wasige, E. (2017) Broadband BowTie Slot Antenna with Tuning Stub for Resonant Tunnelling Diode Oscillators with Novel Configuration for Substrate Effects Suppression. In: European Microwave Conference, London, 3-7 Oct 2016, pp. 421-424. ISBN 9782874870439.

There may be differences between this version and the published version. You are advised to consult the publisher's version if you wish to cite from it.

http://eprints.gla.ac.uk/123127/

Deposited on: 22 August 2016

Enlighten - Research publications by members of the University of Glasgow http://eprints.gla.ac.uk 


\title{
Broadband Bow-Tie Slot Antenna with Tuning Stub for Resonant Tunnelling Diode Oscillators with Novel Configuration for Substrate Effects Suppression
}

\author{
Khalid Hamed Alharbi, Ata Khalid, Afesomeh Ofiare, Jue Wang, and Edward Wasige \\ High Frequency Electronics Group, School of Engineering, University of Glasgow \\ Glasgow, United Kingdom \\ k.alharbi.1@ research.gla.ac.uk; edward.wasige@glasgow.ac.uk
}

\begin{abstract}
Radiation from antennas integrated with InP-based resonant tunnelling diode (RTD) oscillators is usually degraded because of the effects of the large dielectric constant substrate. The common solution has been to use hemispherical lenses to extract the signal from the backside of the substrate. In this paper we present a broadband bow-tie slot antenna with tuning stub which is diced and mounted on a ground plane to alleviate the substrate effects. Here, the large dielectric constant substrate around the antenna conductor is removed. In addition, the ground plane underneath the diced substrate acts as a reflector and, ultimately, the antenna radiates to air-side direction. The antenna was designed and fabricated using photolithography techniques to offer wide bandwidth (return loss $S_{11}<-10 \mathrm{~dB}$ ) between 200-350 $\mathrm{GHz}$ on semi-insulating InP substrate with dielectric constant of $\epsilon_{r}=12.56$. Simulated and measured bandwidth almost extends the frequency range 230-325 $\mathrm{GHz}$. Simulations shows air-side radiation pattern, an antenna gain of around $11 \mathrm{~dB}$ at $290 \mathrm{GHz}$ and $98 \%$ radiation efficiency.
\end{abstract}

Keywords - resonant tunnelling diod; integrated antenna; bow-tie antenna; reflector ground plane; Terahertz

\section{INTRODUCTION}

Terahertz $(\mathrm{THz})$ radiation, whose frequency range lies between millimeter-waves and infrared light in the electromagnetic spectrum has many potential applications in different scientific fields such as medical diagnostics, security imaging, and wireless communication [1-4]. Resonant tunnelling diode (RTD) based $\mathrm{THz}$ oscillators in integrated circuit (IC) form have recently been shown the capability of producing $\mathrm{THz}$ frequencies at room temperature with relatively high power [5-9].

A high performance antenna is a key element by which the $\mathrm{THz}$ radiation can be coupled into free space. An antenna with high gain and directivity is preferable in order to radiate most of the RF power into specific direction and offer larger distance coverage. However, there are a number of challenges when designing antennas on large dielectric constant substrate (such as InP substrate for RTD-based oscillators). Antennas on such

This work was supported in part by the Engineering and Physical Sciences Research Council (EPSRC) of the UK, grant number EP/J019747/1, and in part by the European Commission, grant agreement no. 645369 (iBROW project) substrates tend to radiate most of their energy into and through the substrate [10]. For example, an infinitesimal planar dipole radiates approximately $\varepsilon^{3 / 2}$ more power into the substrate than into free space. In addition, any radiation into the substrate at angles greater than the critical angle $\left(\theta_{c}=\sin ^{-1}\left(\varepsilon r^{-1 / 2}\right)\right)$ are totally internally reflected at the top and bottom surfaces and leading to confinement of the energy inside the substrate as illustrated in Fig. 1. For InP $\left(\varepsilon_{\mathrm{r}}=12.56\right)$ the critical angle is around 16.4 degrees. Theses confined radiation propagates inside the substrate without getting radiated unless a discontinuity appears at the edges leading to degradation in the radiation pattern and antenna performance in general. A practical and common solution to this problem has been to use a hemispherical lens on the backside to collect the power from the bottom side of the substrate [11]. In this paper, instead of using bulky lenses, we propose a broadband bow-tie slot antenna with tuning stub which is diced and mounted on a ground plane for InP-based RTD oscillators. By limiting the substrate area around the antenna conductor, the substrate effects can be reduced. Furthermore, the ground plane underneath the die acts as reflector to re-direct the energy into air direction perpendicular to the substrate.

The rest of the paper is organised as follows. Section II presents the bow-tie antenna used in the proposed concept. The effects of the substrate size are analysed and discussed. The advantages of the proposed diced and grounded antenna are presented in this section. Section III presents the simulation and measurement results of the proposed antenna. Conclusion and future work are provided in sections IV.

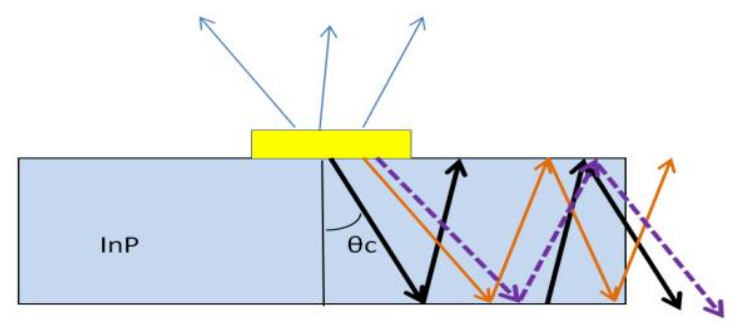

Fig. 1. Illustration of the critical angle and radiation of antenna on-wafer. 


\section{BOW-TIE ANTENNA WITH TUNING STUB}

\section{A. Antenna Design}

A bow-tie antenna is a simple version of planar slot antennas which can offer large bandwidth. A simple CPW-fed bow-tie antenna with tuning stub for increased bandwidth adopted in this work was proposed in Ref. [12]. Fig. 2 shows the geometry of the design. In this paper the antenna was designed for bandwidth between $200 \mathrm{GHz}$ and $350 \mathrm{GHz}$ following the design procedure described in Ref. [12]. Therefore, the antenna dimensions are: $W=840 \mu \mathrm{m}, L=556.5$ $\mu \mathrm{m}, W_{l}=660 \mu \mathrm{m}, W_{2}=600 \mu \mathrm{m}, L_{1}=495 \mu \mathrm{m}, L_{2}=45 \mu \mathrm{m}, L_{3}=$ $77 \mu \mathrm{m}$, and $L_{4}=96 \mu \mathrm{m}$. The CPW line with signal line width of $20 \mu \mathrm{m}$ and gap of $13.8 \mu \mathrm{m}$ to the reflectors sides were calculated to give $50 \Omega$ characteristic impedance. The conducting element is made of $0.4 \mu \mathrm{m}$ thick gold and patterned on the top of the InP substrate.

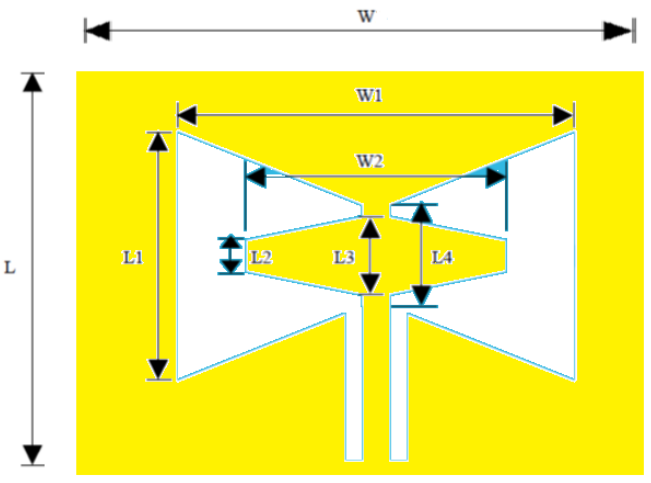

Fig. 2. The geometry of the adopted bow-tie antenna [12].

\section{B. Effects of the Large Dielectric Constant Substrate}

As discussed above, for $\operatorname{InP}\left(\varepsilon_{\mathrm{r}}=12.56\right)$ the critical angle is around 16.4 degrees. Therefore, any radiation into the substrate at angles greater than this angle will be confined inside the substrate. To visualise these trapped energy, the bow-tie antenna was simulated on large area InP (Fig. 3) using HFSS simulator. Fig. 4 and Fig. 5 show the electric and magnetic fields inside the substrate, respectively. These trapped fields seem to lead to the inevitable degradation of the antenna performance when using a planar antenna on a large area substrate of large dielectric constant as shown in the simulated radiation pattern in Fig. 6.

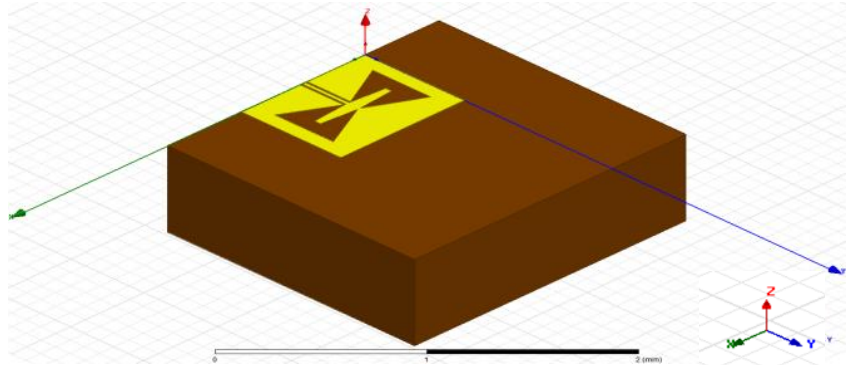

Fig. 3. Antenna geometry on relatively big size substrate used to visualise the fields inside the substrate.
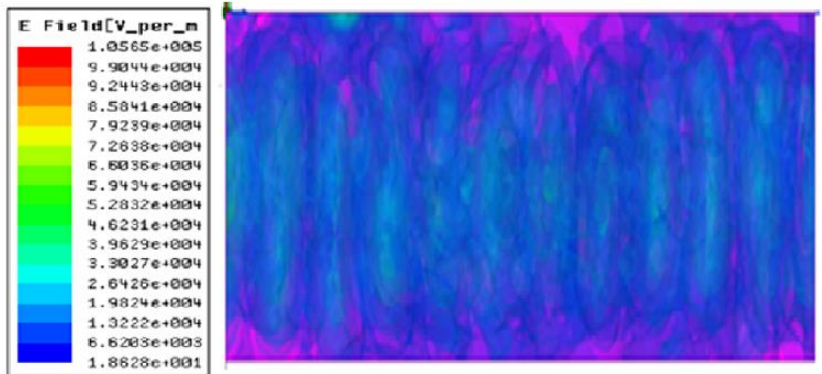

Fig. 4. E-field inside the substrate (side view, yz-plane).
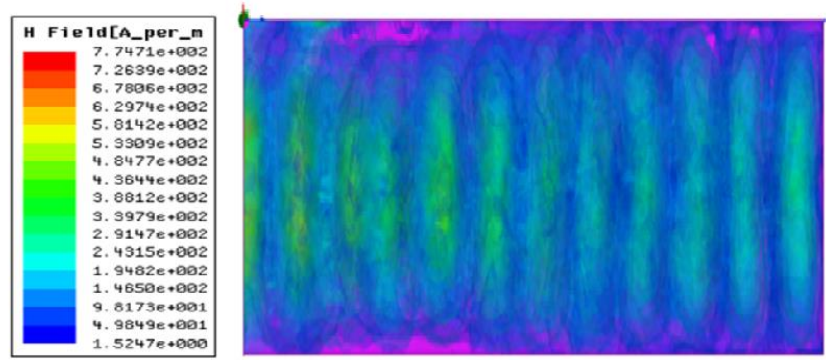

Fig. 5. H-field inside the substrate (side view, yz-plane).

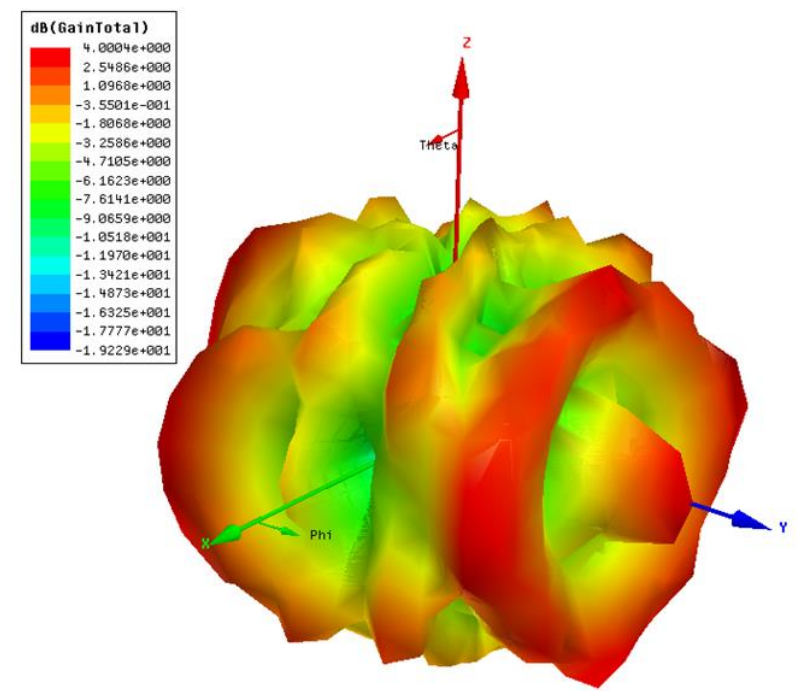

Fig. 6. Radiation pattern of the bow-tie antenna configuration placed on big InP substrate

\section{Diced and Grounded Antenna}

The effects of the substrate can be alleviated if the substrate material around the antenna conductors was removed. This makes the size of the substrate the same as the outer dimension of the antenna. Therefore, the path of confined radiation will be shorter. Furthermore, placing this diced antenna on big size reflector ground plane will enhance the radiation pattern by re-directing any radiation into the substrate direction into air-side perpendicular to the reflector ground plane. Fig. 7 illustrates these radiations from the proposed configuration.

The bow-tie antenna with a tuning stub was fabricated using electron beam lithography process. Electron beam resist 
PMMA was used. The spun PMMA on the InP sample was baked for 3 minutes at $160^{\circ} \mathrm{C}$, followed by e-beam pattern writing using Vestec VB6 beam writer. After development in 1:1 IPA:MIKB, Ti/Au (20nm/400nm) metal scheme was deposited using an electron beam evaporator followed by liftoff in acetone. The antenna was then diced and mounted on big size reflector ground plane. Fig. 8 shows the fabricated antenna.

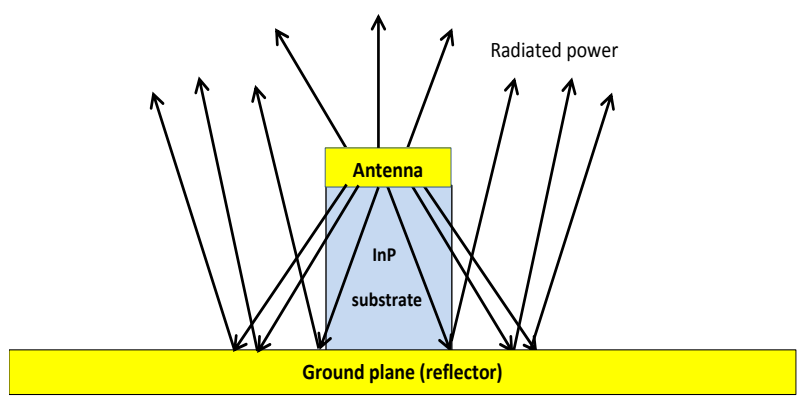

Fig. 7. Illustration of the radiation of diced antenna placed on reflector ground plane.

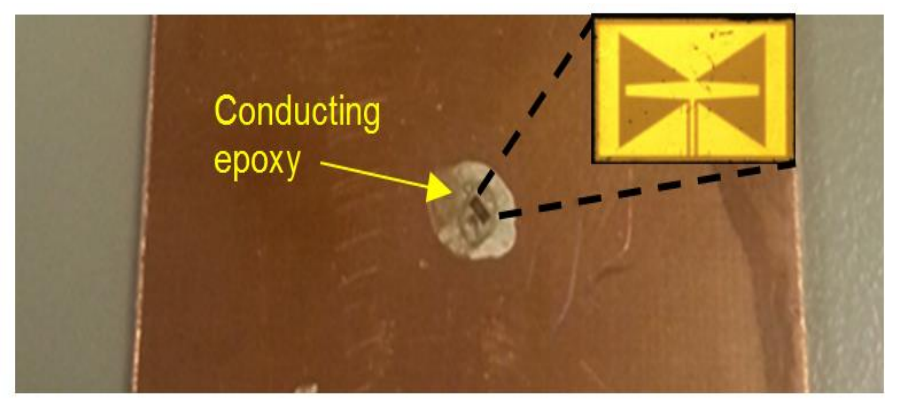

Fig. 8: Fabricated diced antenna placed on reflector ground plane.

\section{RESULTS}

\section{A. Return Loss}

The fabricated antenna (Fig. 8) was measured using a Jband (220-325 GHz) Cascade Microtech millimeter wave vector network analyzer (VNA) with G-S-G infinity probes. The VNA was calibrated for 2-port S-parameter measurements using the short-open-load-thru (SOLT) two port calibration process on a Cascade impedance substrate standard. Fig. 9 shows the measured and simulated return loss of the designed antenna. The bandwidth extends almost the entire frequency band from 220-325 GHz. The discrepancy between the measured and simulated results can be attributed to unwanted signal reflections from surrounding objects during measurements.

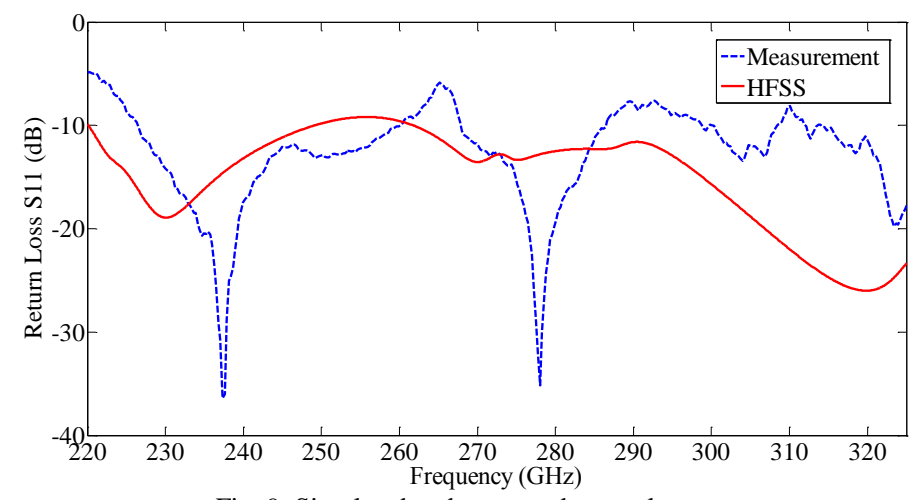

Fig. 9. Simulated and measured return loss.

\section{B. Radiation pattern}

The simulated 3D radiation pattern of the antenna at 290 $\mathrm{GHz}$ is shown in Fig. 10. The proposed antenna radiates upward toward the air side (perpendicular to the substrate) which proves the advantages of both the diced antenna and the reflector ground plane which is placed on the bottom side of the InP substrate. The antenna gain is around $11 \mathrm{~dB}$ and the radiation efficiency is $98 \%$.

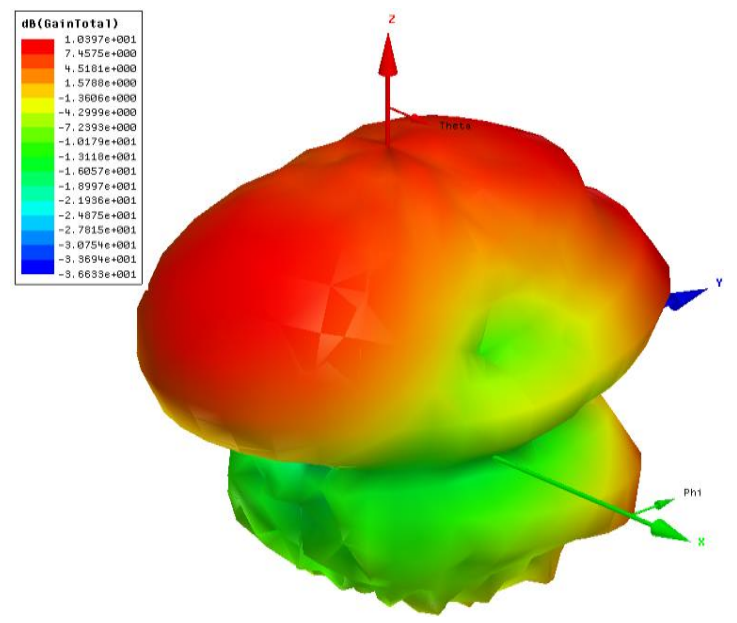

Fig. 10. Simulated 3-D radiation pattern.

\section{Two Port Bow-TIE SLOT ANTENNA}

A two port bow-tie slot antenna was fabricated and characterized to investigate the suitability of integration the antenna with the power combining RTD oscillator circuit which has been proposed by the group recently [13]. The oscillator circuit is based on mutual coupling of two individual oscillators and deliver the output power to a single load. The oscillator can employ up to four RTD devices for higher power operation. Output power of $1.1 \mathrm{~mW}$ at $\sim 310 \mathrm{GHz}$ was obtained from this circuit and results will be published elsewhere soon. The antenna was designed with the same dimensions presented above but with two CPW feed lines. Fig.11 shows the fabricated two port bow-tie antenna. 


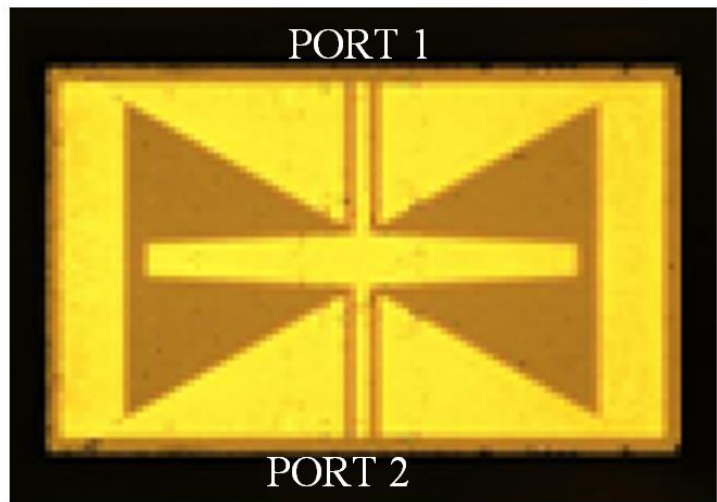

Fig. 11. Fabricated Bow-tie antenna with two CPW feed lines.

The measured return loss $\left(\mathrm{S}_{11}\right.$ and $\left.\mathrm{S}_{22}\right)$ of the fabricated two port antenna are shown in Fig. 12, while Fig. 13 shows the measured insertion loss $\left(S_{21}\right.$ and $\left.S_{12}\right)$. All the 4 S-parameters indicate that most of the signal send towards the antenna is radiated, and little is reflected back or transmitted through to the other port. Compared with the return loss obtained from one port antenna in Fig. 9, the return loss from the two ports are similar in general with some discrepancy that might be due to the measurement environment, e.g. close proximity of the pair of probes and reflections. Thus, the two port bow-tie antenna is a good candidate to be integrated with the coupled oscillator circuit that employs four RTD devices for high power operation.

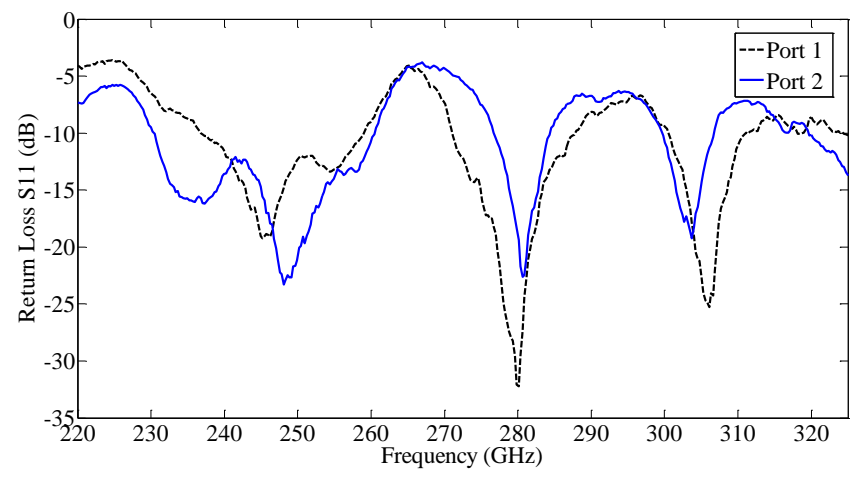

Fig. 12. Return loss $\left(\mathrm{S}_{11}\right.$ and $\left.\mathrm{S}_{22}\right)$ of the two port bow-tie antenna with stub.

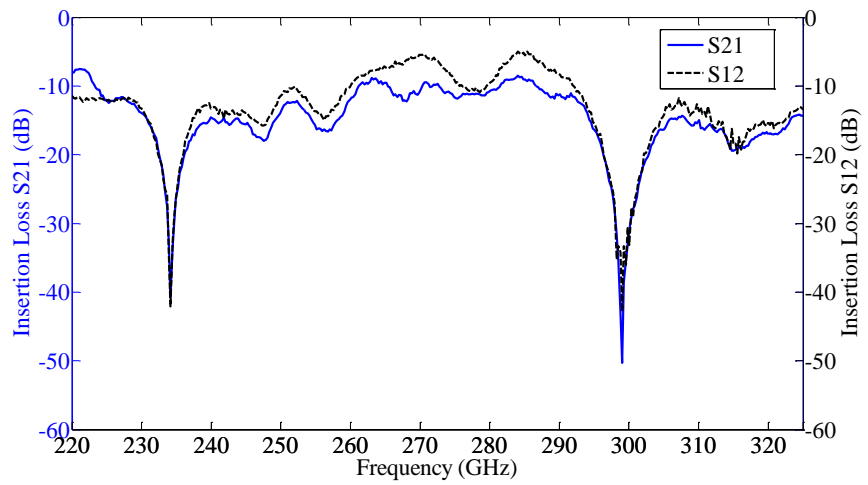

Fig. 13. Insertion loss $\left(S_{21}\right.$ and $\left.S_{12}\right)$ of the two port bow-tie antenna with stub.

\section{CONCLUSIONS}

In this paper, a grounded and diced bow-tie slot antenna with tuning stub for terahertz applications was presented. It offers a known input impedance and wide bandwidth. The diced and grounded antenna suppresses the substrate effects and radiates into the air perpendicular to the substrate. Future work is focused on integrating this antenna with InP-based RTD THz oscillators.

\section{REFERENCES}

[1] P. H. Siegel, "Terahertz technology," IEEE Transactions on Microwave Theory and Techniques, vol.50, no.3 Mar 2002, pp.910-928.

[2] M. Tonouchi, "Cutting-edge terahertz technology," Nature Photonics, vol. 1, 2007, pp. 97-105.

[3] T. Kleine-Ostmann, and T. Nagatsuma, "A review on terahertz communications research.," Journal of Infrared, Millimeter, and Terahertz Waves, vol. 32., 2011, pp. 143-171.

[4] H. B. Liu, H. Zhon, N. Karpowicz, Y. Chen, and X. C. Zhang, "Terahertz spectroscopy and imaging for defense and security applications," Proceedings of the IEEE, vol. 95, 2007.pp. 1514-1527.

[5] J. Wang, K. Alharbi, A. Ofiare, H. Zhou, Ata Khalid, D. Cumming, and E. Wasige, "High Performance Resonant Tunneling Diode Oscillators for THz Applications," in Compound Semiconductor Integrated Circuit Symposium (CSICS), 2015 IEEE , New Orleans, LA, USA, 11-14 Oct.2015, pp.1-4

[6] T. Maekawa, H. Kanaya, S. Suzuki, M. Asada, “Frequency increase in terahertz oscillation of resonant tunnelling diode up to $1.55 \mathrm{THz}$ by reduced slot-antenna length," Electronics Letters , vol.50, no.17, 2014 , pp.1214-1216.

[7] S. Suzuki, K. Hanashima, N. Kishimoto, and M. Asada, "Sub-THz resonant tunneling diode oscillators with offset-fed slot antenna," in International Conference on Indium Phosphide \& Related Materials, Matsue, Japan, 2007, pp. 530-533.

[8] K. Hinata, M. Shiraishi, S. Suzuki, and M. Asada, "High power THz oscillators with offset-fed slot antenna and high current density resonant tunneling diodes," in International Conference on Infrared, Millimeter, and Terahertz Waves, Busan, 2009, pp. 1-2.

[9] S. Suzuki, K. Hinata, M. Shiraishi, M. Asada, H. Sugiyama, and H. Yokoyama, "RTD oscillators at $430-460 \mathrm{GHz}$ with high output power $(\sim 200 \mu \mathrm{W})$ using integrated offset slot antennas," in International Conference on Indium Phosphide Related Materials, Kagawa, 2010.

[10] E. R. Brown, C. D. Parker and E. Yablonovitch, "Radiation properties of a planar antenna on a photonic-crystal substrate," Journal of the Optical Society of America B, vol. 10, 1993, pp. 404-407.

[11] N. Orihashi, S. Suzuki, and M. Asada, "One THz harmonic oscillation of resonant tunneling diodes," Applied Physics Letters, vol. 87, 2005, pp. 233501-1 -233501-3.

[12] A. A. Eldek, A.Z. Elsherbeni, and C. E. Smith. "Characteristics of bowtie slot antenna with tapered tuning stubs for wideband operation." Progress In Electromagnetics Research 49 (2004): pp.53-69.

[13] A. Ofiare, J. Wang, K. Alharbi, A. Khalid, E. Wasige and L. Wang, "Novel tunnel diode oscillator power combining circuit topology based on synchronisation," Asia-Pacific Microwave Conference (APMC), Nanjing, 2015, pp. 1-3. 
